\title{
HLA-Identical Dendritic-Leukemic Cell Hybrids Generate Specific CTLs in Vitro
}

\author{
Rinat Eshel ${ }^{1 *}$, Meirav Shpringer ${ }^{1}$, Nadia Voskobinik ${ }^{2}$, Akiva Vexler ${ }^{3}$, Rami Ben-Yosef $^{3}$, Inna Fabian ${ }^{4}$, \\ Boris Tartakovsky ${ }^{1}$, Elizabeth Naparstek ${ }^{1,4}$
}

${ }^{1}$ Department of Hematology and Bone Marrow Transplantation, Tel-Aviv University, Tel-Aviv, Israel; ${ }^{2}$ Department of Genetics, Tel-Aviv University, Tel-Aviv, Israel; ${ }^{3}$ Department of Oncology, Tel-Aviv University, Tel-Aviv, Israel; ${ }^{4}$ Tel-Aviv Sourasky Medical Center, Tel Aviv and Sackler Faculty of Medicine, Tel-Aviv University, Tel-Aviv, Israel.

Email: rinate@tasmc.health.gov.il

Received April 19 ${ }^{\text {th }}, 2010$; revised May 18 ${ }^{\text {th }}, 2010$; accepted June $18^{\text {th }}, 2010$.

\begin{abstract}
While being instrumental in the treatment of leukemic relapse after allogeneic hematopoietic stem cell transplantation, the impact of donor lymphocyte infusion (DLI) and its effectiveness remain debatable. Consequently it is widely accepted that more efforts are needed in order to make DLI more effective. This communication thus deals with the generation of specific CTLs in the clinical setting of HLA matched hematopoietic stem cell transplantation, to be used as an improved DLI treatment for post-transplantation relapsed leukemias. We assessed the potential of fused dendritic cells from donor origin, with leukemic cells from the HLA matched recipient for the generation of donor anti-tumor CTLs. Leukemic cells and donor dendritic cells were fused using polyethylene glycol (PEG). The hybrids were analyzed for double phenotype of both DC and tumor, and used for the education and generation of cytotoxic donor lymphocytes. Results demonstrate that efficient and specific CTLs can be generated and used in vitro for the elimination of the recipient tumor cells. These results form the basis for the establishment of a novel methodology aimed at generating active or passive anti-leukemic vaccine in relapsed patients.
\end{abstract}

Keywords: Dendritic Cells, Cytotoxicity, Cellular Therapy

\section{Introduction}

Cancer immunotherapy is hampered by the fact that tumor specific antigens are either unknown, non-immunogenic or that the tumor develops means to evade the host immune response [1-3]. In attempting to circumvent these obstacles, novel approaches of cancer immunotherapy based on the development of potent tumor vaccines are extensively explored [4]. The use of dendritic cells (DCs), in that respect, has recently gained a remarkable momentum, both in pre-clinical and clinical settings [58].

Dendritic cells are potent antigen presenting cells (APCs) due to the expression of MHC I/II, co-stimulatory and adhesion molecules that provide secondary signals for the generation of primed T cells [9-11]. The introduction of tumor specific antigens into dendritic cells leading to the generation of cytotoxic T Lymphocytes (CTLs) against malignant cells has already been proven to be effective $[4,12]$. Thus, DCs can be pulsed with liposomal DNA, tumor cells or apoptotic cell fragments, peptides eluted from tumor cells, membranes, lysates, RNA and more [13-18]. Such strategies have generated positive in vitro immune responses directed against the relevant tumor cells. Nevertheless a more promising approach to the induction of primary anti-tumor immunity is through the generation of fusion between tumor cells and DCs $[13,6]$ that use primarily whole tumor cells as the source of pulsing antigen. Indeed, cancer immunotherapy by DC/ tumor cell fusion hybrids using solid carcinoma cells, in mouse models or in vitro with human cells such as breast and ovarian carcinoma, has been shown to elicit potent anti tumor effects via the induction of immune responses against multiple tumor-associated antigens [14,19-21].

The therapeutic potential of tumor-DC fused cells has been tested in phase I/II clinical trials in patients with melanoma and breast cancer $[19,22]$. The results demonstrated the safety of the vaccine although the therapeutic efficacy of such a procedure was shown to be variable but promising.

Immunotherapy of hematological malignancies such as myeloid leukemias is of particular complexity since very 
few tumor antigens are known in those tumors [6,23]. Presently, the most clinically meaningful immunotherapeutic modality in hematological malignancies remains allogeneic stem cell transplantation followed, upon relapse, with the administration of allogeneic, donor derived $\mathrm{T}$ lymphocytes (DLI), aiming to generate a clinically significant graft-versus-leukemia (GVL) response [24-31]. Although effective in the chronic phase of chronic myelogenous leukemia (CML), DLIs have been of limited effectiveness in patients with other hematological malignancies, such as ALL principally due to the high proliferative rate of the tumor cells and their relative resistance to immunotherapy. Hence, additional approaches to improve DLI effectiveness are necessary. These may include the use of immunomodulatory cytokines, exvivo priming of the donor lymphocytes to the relevant tumor antigens and infusion of alloreactive NK cells (reviewed by Soiffer 2008) [32].

The use of DC-tumor hybrids has also been assessed in hematological malignancies, holding promise as a cellular vaccine for some of them [33,7,34]. For example, the fusion of AML myeloblasts and autologous DCs [7] or myeloma cells and allogeneic dendritic cells [34], were demonstrated to generate in vitro potent cytotoxic lymphocytes.

Nevertheless, the DC-tumor hybrid approach in HLAmatched allogeneic stem cell transplantation has never been assessed, particularly in the context of post relapse DLI treatment. It is expected that such a procedure may lead to the induction of very potent anti tumor CTLs selected in vitro for no-GVH activity. In addition, these fused cells could also serve as active and potent cell vaccine for pre-emptive immunization of patients at high risk for relapse. These issues represent the subject matter of our research and communication.

\section{Materials \& Methods}

The study was approved by the Institutional Helsinki committee, and appropriate informed consents were obtained from all patients and healthy donors.

\section{Collection of cell samples}

Leukemia cell samples from blood or bone marrow were obtained from newly diagnosed patients with acute leukemia treated in our department. Peripheral blood lymphocytes were collected form their prospective matched HLA donors by direct venipucture or collected by lympho-pheresis (COBE Spectra, COB BCT, Lakewood, CO USA).

Mononuclear fractions were isolated using Ficoll-Hypaque (FH) separation, washed and cryopreserved in liquid nitrogen (using 10\% DMSO as cryo-protectant), according to the standard method. If needed, a positive selection of leukemic cells with the relevant membrane marker beads (such as CD34) was performed in order to obtain a pure population of leukemic cells.

\section{Preparation of immature DCs in vitro}

Immature donor dendritic cells were derived from the mononuclear fraction by magnetic beads purification of CD14 positive cells using the Miltenyi MS-MACS isolation column (Miltenyi Biotec, Bergisch Gladbach, Germany). This enriched population was assessed for purity using standard flow-cytometry technique. Purified monocytes were cultured in 6 well plates (TPP, Zollikofen, Switzerland), $2 \times 10^{6}$ cells/3 ml RPMI 1640 supplemented with $1 \%$ L-Glutamine, $1 \%$ antibiotics mix (Penstrep, Biological Industries, Bet-Haemek, Israel), 5\% autologous serum, GM-CSF (200 units/ml) and IL-4 (500 units/ml) (R\&D Systems, Minneapolis, USA), for 7 days. Half feeding with cytokines was performed every two days. The DCs collected from these cultures following the induction with the cytokine mixture, were further analyzed by flow-cytometry using markers characteristic of DC (see below).

\section{Preparation of DC-leukemia cell hybrids}

Dendritic - leukemia cell hybrids were generated by fusion as described by [20] using the chemical membrane-destabilizing polyethylene glycol (PEG, Roche Diagnostics, Mannheim, Germany). Cells were mixed at $1: 1$ ratio in $2 \mathrm{ml}$ serum free RPMI 1640 in the presence of $50 \%$ PEG, for $5 \mathrm{~min}$ at room temperature. Cells were washed in serum free medium, resuspended in culture medium composed of RPMI 1640 supplemented with $10 \%$ heat inactivated FCS, $1 \%$ L-Glutamine, $1 \%$ antibiotics mix, $500 \mathrm{u} / \mathrm{ml}$ GM-CSF and cultured for 7 days in 6 well plates.

\section{Maturation of DC-leukemia hybrid}

Fused DC-tumor cells were subjected to maturation using a mixture of cytokines: TNF- $\alpha$ (100 ng/ml), IL-6 (1 $\mu \mathrm{g} / \mathrm{ml}), \mathrm{IL}-1 \beta(50 \mathrm{ng} / \mathrm{ml})$ (Peprotech, New Jersey, USA) and PGE2 (1 $\mu \mathrm{g} / \mathrm{ml}$, Cayman Chemical, Michigan, USA) for 3 days in culture medium. Maturation was assessed by flow-cytometry using the specific mature DC marker CD83 (Becton Dickinson, San Jose, CA, USA). After maturation, the fused cells were irradiated (3000 cGy) and used as stimulators for the generation of the specific CTLs. In addition dendritic cells alone were cultured in the presence of the same cytokines and used as controls.

\section{Phenotype analysis}

$2 \times 10^{5}$ cells were used for phenotypic analysis using fluorescein isothiocyanate (FITC)-conjugated monoclonal antibodies (mAbs) against CD86, major histocompatibility complex (MHC) class I, II, and phycoerythrin (PE)-conjugated mAbs against CD14 (DAKO, Glostrup Denmark) and CD83 (Becton Dickinson, San Jose, CA, USA). For tumor cells and DC-tumor hybrids analysis and CTL characterization, cells were stained respectively 
with mAbs against CD15, CD13 (DAKO) and CD34 (Becton Dickinson, San Jose, CA, USA), or with anti CD3, CD4 and CD8 (DAKO). Cells were incubated with antibodies for $30 \mathrm{~min}$ at $4^{\circ} \mathrm{C}$, washed in phosphate-buffered saline (PBS, Bet Haemek) and resuspended in 300 $1 \mathrm{PBS} /$ sodium azide $(0.02 \%$, Sigma Aldrich, Steinheim, Germany) for analysis using the FACSCalibur flow-cytometer system (Becton Dickinson, San Jose, CA, USA). Appropriate isotype-matched control mAbs were also used.

\section{Fusion analysis}

\section{Fluorescent dye staining}

The immature DCs and leukemic cells were labeled with membrane fluorescent dye PKH 67 (green fluorophore) and fluorescent dye PKH 26 (red fluorophore) respectively, purchased from Sigma (Aldrich, Steinheim, Germany). The two cell populations were then fused as detailed above. After fusion, the cells were assessed for fusion efficiency by flow-cytometry. Double positive cells were recorded as hybrids. In addition, fluorescence microscopy analysis was performed on stained DC and tumor cells, as well as on the hybrids. The appearance of yellow fluorescence was characteristic of fused cells (Olympus BX52 fluorescence microscope equipped with green, red and Tric filters).

Cytogenetic FISH analysis

FISH analysis was performed with a Vysis ${ }^{\circledR}$ LSI $^{\circledR}$ BCR/ABL Dual Color Translocation Probe (VYSIS, Downers Grove, Illinois U.S.A.). The BCR/ABL probe is a mixture of the LSI ABL probe labeled with SpectrumOrange and a BCR probe labeled with spectrumGreen. FISH was performed according to the manufacturer's instructions (VYSIS Inc.).

\section{Generation of CTLs}

Donor peripheral blood lymphocytes were purified using FH gradient and added to irradiated DCs-leukemia hybrids at a ratio of $3: 1$.

As a control, the lymphocytes were co-cultured with the irradiated unfused DCs and irradiated tumor cells alone, for 21-24 days. After one week of co-culture and every two days thereafter recombinant IL-2 $(20 \mathrm{u} / \mathrm{ml})$ (R\&D Systems, Inc. Minneapolis, USA) was added to all cultures. In addition, same amount of stimulatory tumor cells, DCs and hybrids were added once a week.

CTL activity and specificity assessment

CTLs derived from the different co-cultures as well as unstimulated donor PBLs were tested for their cytotoxicity against the patient leukemic cells or third party specificity control tumor cells, using the Lacto-dehydrogenase (LDH) cytotoxicity assay (LDH Cytotoxicity Detection Kit, Roche, Penzberg, Germany) in several effector:target cell ratios.

Briefly, $5 \times 10^{4}$ tumor cells were incubated in a 96- well flat bottom plates with the effectors at different ratios for 4 hours at $37^{\circ} \mathrm{C} 5 \% \mathrm{CO}_{2}$. Then, the cells were processed as instructed by the manufacturer. Controls included medium, CTLs and tumor target cells for spontaneous and maximal release of LDH.

\section{Results}

Peripheral blood or bone marrow samples containing at least $90 \%$ leukemic cells were obtained from newly diagnosed AML and ALL patients. Tumor cells were detected primarily as an abnormal population upon CD45-side scatter FACS analysis. The gated tumor population was further analyzed for specific membrane markers such as CD15, CD7, CD19, CD34, and CD13, prior to freezing.

Peripheral mononuclear cells (MNC) from the prospective HLA-matched (A, B, C, DR and DQ) donors were frozen prior to any transplantation-related manipulation. These cells were used both a source of dendritic cells and of future CTLs.

\subsection{Isolation and Purification of DCs}

CD14 positive monocytes, positively selected by immunomagnetic beads from the mononuclear cells of the prospective selected donor, were cultured in the presence of human IL-4 and GM-CSF for 7 days. The immature DC's thus generated, were analyzed by flow cytometry for specific membrane markers. Results shown in Figure $\mathbf{1}$, demonstrate one representative experiment in which the membrane markers typical to immature DC's, i.e., high expression of CD1a, CD86 and HLA, together with lack of CD14 and CD83 was evident.

\subsection{Hybrids Generation}

The tumor cells were thawed and assessed for viability and number as detailed in $\mathrm{M} \& \mathrm{M}$.

Next, tumor cells and donor immature DC were mixed at 1:1 ratio in the presence of PEG for $5 \mathrm{~min}$. Prior to mixing, a small number of cells were stained with membrane fluorescent dyes, the DC in green (PKH2) and the tumor cells in red (PKH26), in order to assess proper fusion, as detailed below. Fusion of these cells was performed separately, under same conditions.

Cells were then washed and cultured for 7 days in the presence of GM-CSF. Fusion efficacy was assessed according to several parameters.

Validation of fusion

Detailed below are several methods utilized to confirm the fusion of the dendritic and leukemia cells.

Flow cytometry

Double staining of membrane fluorescent dye, PKH2 and PKH26 by flow cytometry: A representative experiment is shown in Figure 2(a) in which about 35\% of the analyzed cells were found to express both colors. In 


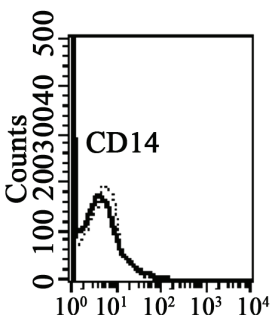

CD 14 APC

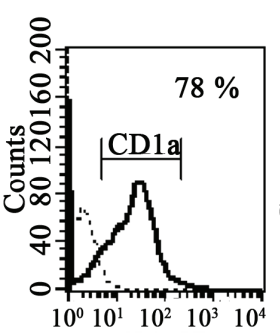

CD1a PE
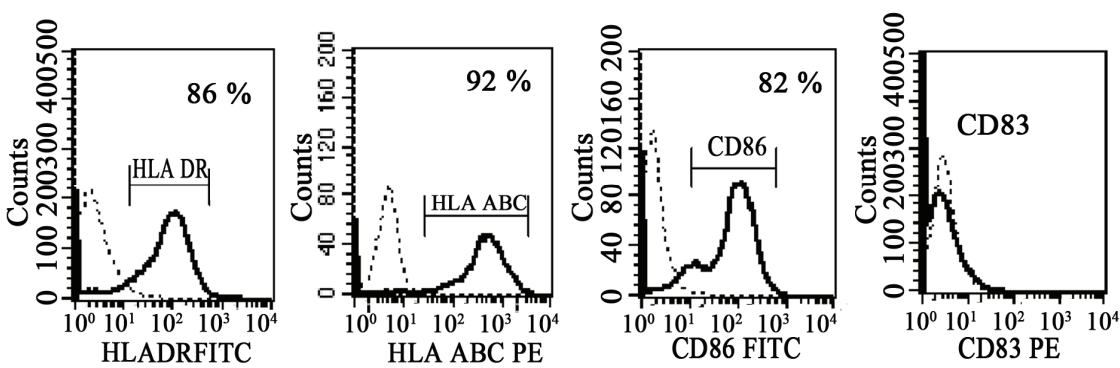

Figure 1. Phenotype analysis of dendritic cells: FACS analysis of dendritic cell surface markers using specific monoclonal antibodies (bold line) and isotype controls (dot line). Immature dendritic cells obtained following cytokine induction for 7 days are shown. Histograms demonstrate negative expression of CD14 and CD83 and positive expression of CD1a, CD86, HLA class I and II.
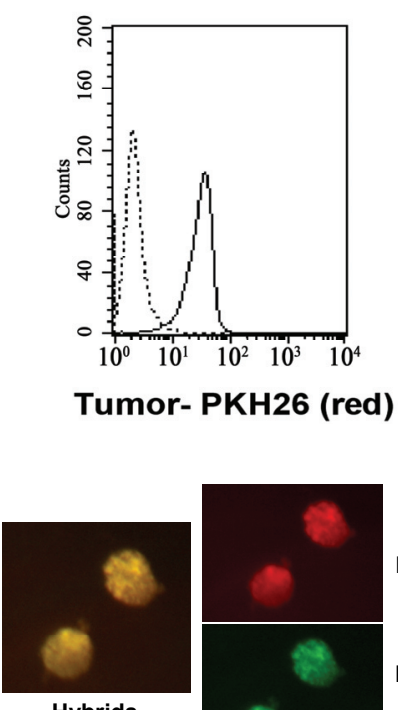

Hybrids

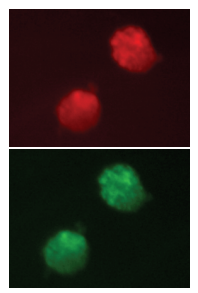

(b)

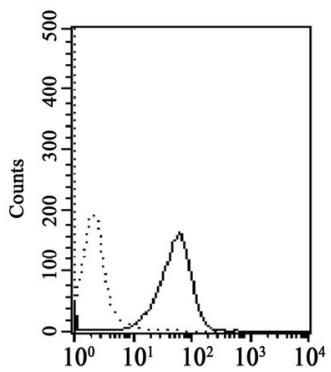

DC- PKH2 (green)

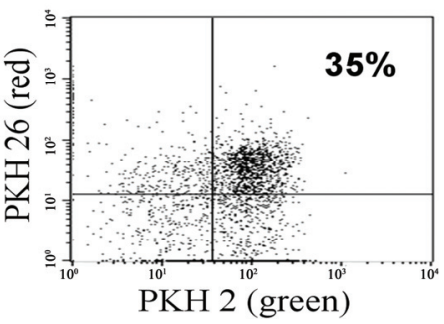

(a)
Leukemic cells

Donor's DCs

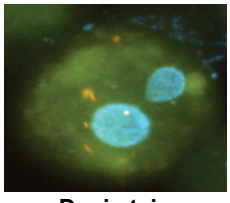

Dapi stain

(c)

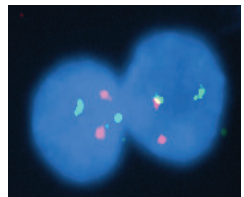

(d)

Figure 2. Validation of fusion between leukemic cells and normal HLA matched DCs. (a) Assessment of fusion by immunofluorescent membrane dye. Leukemic cells from a $\mathrm{Ph}^{+} \mathrm{ALL}$ patient stained with $\mathrm{PKH} 26$ red fluorophore (left histogram) and DCs from his matched donor stained with PKH2 green fluorophore (right histogram). Bold lines represent the stained cells; dot lines histograms represent unstained cells. Dot plot analysis on the right panel shows double positive PEG induced hybrids. As shown, $35 \%$ of the cells in the mixed culture consisted of dendritic-tumor hybrids. Comparable results were obtained using AML cells; (b) Fluorescence microscopy of the DC-tumor hybrid cells: Microscope (Olympus BX52 fluorescence microscope) picture of cells from Figure 2(a) above. Same field was analyzed under 3 different filters (green, red and TRITC U-M61002). Upper right: red filter showing PKH26 stained tumor membrane, lower right: green filter showing PKH2 stained dendritic cell membrane. Left picture: TRITC filter showing the yellow combination of the hybrid membraneIdentical results were obtained using AML and ALL cells; (c) Fluorescence microscopy of an ALL-DC hybrid. Figure 2(c) demonstrates one cytoplasm containing two different nuclei visualized by Dapi stain. Identical results were obtained with AML cells. (d) FISH analysis of $\mathrm{Ph}^{+}$ALL-DC fused cells: Chromosome 9 specific ABL probe (red-orange fluorescence) and chromosome 22 BCR specific probe (green fluorescence) were used in order to visualize the chromosomes and the 9; 22 translocation (yellow fluorescence). Note normal cytogenetic picture on the left nucleus showing two 9 and two 22 chromosomes, while the right nucleus demonstrates the yellow 9; 22 translocation and one separate chromosome 9 and one separate chromosome 22.

about five consecutive experiments an average of $36 \%$ of the cells were found to be double positive as statistically expected from a 1:1 (DC-tumor) ratio. A fluorescence microscopy of the fused cells was also conducted. This is shown in Figure 2(b), in which the red and green fluo- rescence are demonstrated to yield a yellow light emission in presumably fused hybrid cells.

The tumor-fused DC cells were induced to mature using a cocktail of TNF, IL1-, IL-6, PGE2, GM-CSF and IL-4, for 2 days. The maturation of the dendritic tumor 
fused cells was validated by the appearance of CD83. Occasionally, the co-expression of CD83 and of a tumor aberrant membrane marker such as CD15 or CD13 was also monitored by FACS analysis (data not shown).

\section{Cytogenetic FISH analysis}

Cells from the fused cultures were applied on microscope slides and stained with DAPI in order to visualize the nuclei. Numerous cells containing two nuclei were readily detected in the cultures as shown in Figure 2(c). These experiments were performed with both ALL and AML cells with comparable efficiency and success. In order to identify the origin of the nuclei in the fused cells other slides were processed for FISH analysis as described above. Note that cytoplasm can not be visualized under the FISH staining protocol.

FISH based cytogenetic analysis was performed making use of the BCR-ABL translocation in $\mathrm{Ph}^{+}$ALL patients. This is demonstrated in Figure 2(d). The picture demonstrates a fused cell that contained two nuclei: one nucleus containing two chromosomes 9 (red color) and 2 chromosomes 22 (green color); the second nucleus containing one chromosome 9 , one chromosome 22 and a translocated 9-22 chromosome (yellow color).

\subsection{CTL Generation}

At the end of the maturation process, the tumor-DC fused cells were co-cultured with the donor mononuclear cells at a ratio of 10:1 MNCs: DC-tumor hybrid cells for 21-24 days. As controls, donor's mononuclear cells were also co cultured with autologous mature DC's alone and with the tumor cells alone (same tumor used for the fusion).

At the end of the incubation period, educated mononuclear cells were collected, washed and analyzed for cell phenotype. Cells were stained for membrane expression of CD3, CD4, and CD8. As shown in the left panel of Figure 3 (representative experiment), most of the CD3 positive lymphocytes did not survive in the DC and tumor cultures and only about $20-30 \%$ of those cultures were CD3 positive cells. It should be noted that in these two groups, cell viability was very poor in comparison to Mononuclear cells were collected and stained for CD3APC, CD4-PE and CD8-FITC and appropriate isotype

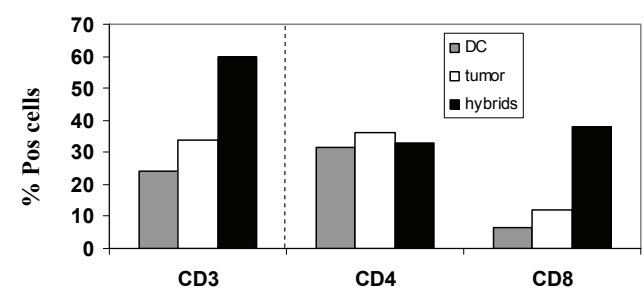

Figure 3. Phenotype analysis of mononuclear cells cocultured with dendritic cells, AML leukemic cells or DCtumor hybrids for 21 days. controls. Cells were analyzed by FACS immunofluorescence. Bars represent the ratio of $\mathrm{CD} 3$ positive cells from the total number of cells in the cultures (left) and the percent of CD4 and CD8 positive cells from the CD3 positive ones in the various groups (middle and right).the hybrid group. Moreover, in the hybrid group $60 \%$ of the cells were $\mathrm{CD} 3$ positive and in this co-culture, a preferential induction of CD3 CD8 double positive cells was observed whereas $\mathrm{CD} 3$ positive $\mathrm{CD} 4$ positive cells were found in equal proportion in the three groups.

As shown, in the DC and tumor groups, most of the $\mathrm{CD} 3$ positive cells detected were not $\mathrm{CD} 8$ positive cells Thus, CD3 CD8 double positive cells, characteristic to CTL phenotype, were generated exclusively in co-cultures of donors mononuclear cells with the tumor-donor DC hybrids.

\subsection{Cytotoxicity Assays}

The cultured donor CTLs were assayed for cytotoxicity against their relevant AML patient target cells. In preliminary experiments, the ratio of co-culture was calibrated. Educated mononuclear cells were cultured with the tumor cells at various ratios. The cytotoxicity was assessed by LDH release as shown in Figure 4(a). The results demonstrate clearly that the education of the CTLs on the hybrids generated the highest cytotoxic effect and that the optimal killing was observed at the $50: 1$ ratio. This ratio was further used in all coming experiments.

Figure 4(b) demonstrates the percent of tumor cells (3 AML and 1 ALL patients) killed by the educated CTLs in four consecutive experiments, using four different donors and their matched HLA recipient pairs. Up to $32 \%$ of the tumor cells were found to be killed by the CTLs educated on the hybrids as opposed to only $0-15 \%$ or $1-2 \%$ in the controls.

A limited specificity study was initiated with the use of an unrelated tumor of the same histological type (AML). This is shown in Figure 5 in two consecutive experiments. The results clearly demonstrate that the CTLs generated against the relevant tumor cell-DC hybrids were not cross reacting against irrelevant, unrelated leukemia cells. In addition, no cytotoxicity could be detected in the mononuclear cell population prior to education on the DC-tumor hybrids.

\section{Discussion}

The primary massage of this communication concerns the proof of concept related to the in vitro generation of tumor specific CTLs educated on DC-tumor hybrids in an HLA matched setting. This has never been attempted before.

The therapeutic options for patients that relapse with 


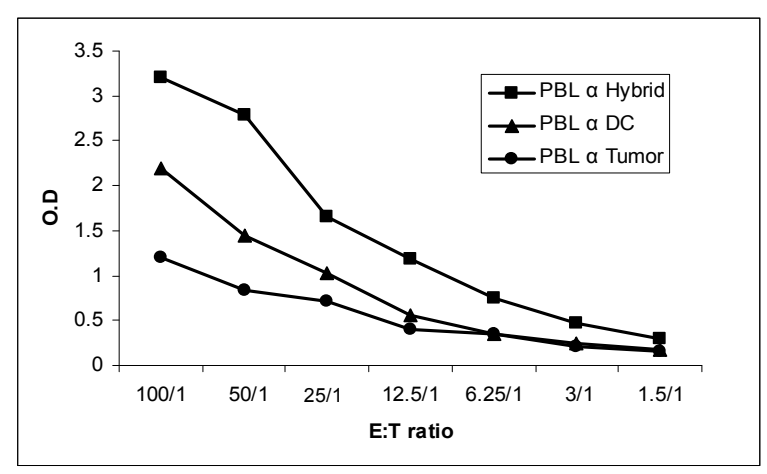

(a)

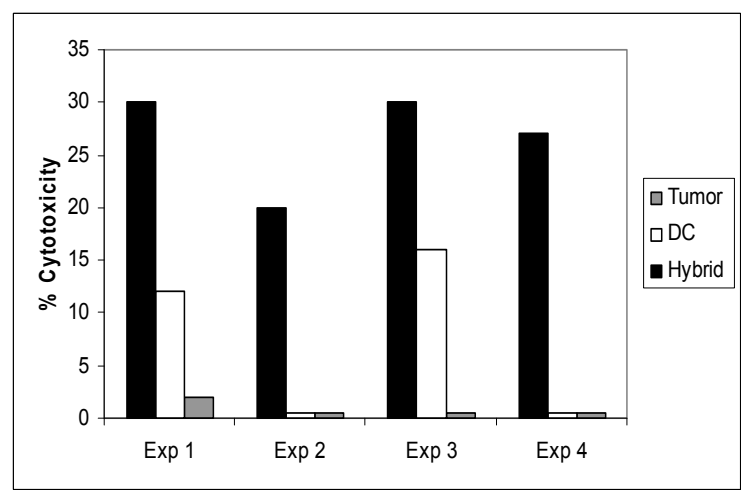

(b)

Figure 4. Cytotoxic activity of HLA-matched donor CTLs. (a) Calibration of LDH release for optimal E:T ratio: CTLs of the donor (originally PBLs) generated against DC, tumor cells or DC-tumor hybrids were assayed for cytotoxicity against tumor cells upon incubation for 4 hours in 96 well plates at $37^{\circ} \mathrm{C}$. At the end of the incubation, cultures were processed as instructed by the manufacturer of the LDH Cytotoxicity Detection Kit. Lines represent O.D. of LDH released at the different ratios in the different groups; (b) Anti tumor cytotoxicity of HLA-matched CTLs: Four separate cytotoxicity assays using four different donor-patient sets at 50:1 E:T ratio, were performed. The killing of the tumor target cells by the CTLs generated on DC, tumor, DC-tumor hybrids was calculated specifically as instructed. Bars represent the percent of tumor cells killed by CTLs in the various groups.

leukemia after allogeneic stem cell transplantation are limited and their overall prognosis is poor. At an early stage of the relapse, cell-mediated immunotherapy with donor lymphocytes (DLI), aimed to induce a potent graft-versus-leukemia (GVL) effect, is most commonly used [25,28,29,35-37]. This methodology however, has certain significant drawbacks. First, the immune cells injected are naïve and therefore are expected to undergo tumor priming in vivo. This process may not be sufficiently efficient and the leukemic process may outgrow the GVL effects. Second, the in vivo priming may not be restricted to the tumor antigens only. Indeed, an unde-

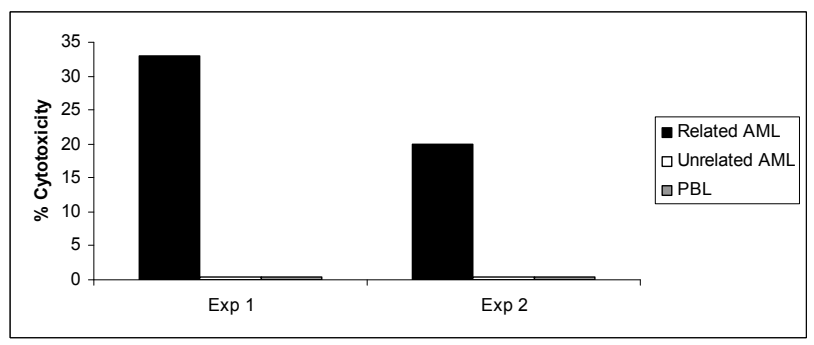

Figure 5. Specificity of hybrid-stimulated CTLs. CTLs were generated on DC-AML hybrids and assayed for cytotoxicity on the same AML target cells used for the hybrid generation, or AML cells derived from a different patient (HLA unmatched). Unstimulated PBLs (designated in the figure as PBL) from the same donors were assayed for cytotoxicity in the same experiments. Two different donor-patient experiments are shown. (Cytotoxic assay as described in legend to Figure 4).

sirable graft versus host $(\mathrm{GVH})$ response is often observed after DLI $[38,39]$. Third, the donor lymphocytes infused, not being properly manipulated, may also contain regulatory, suppressor cells [40]. Several investigators have indeed tried to manipulate the DLI prior to its infusion for better and more specific anti-leukemic effect. For example, depletion of CD8 positive cells [41,42], activation of donor $\mathrm{T}$ cells with anti-CD3 and anti-CD28 coated beads [43], ex vivo insertion of suicide genes into donor T cells prior to DLI [44]. Others have used donor NK cells alloreactivity in matched and mismatched hematopoietic transplants $[45,46]$. Accordingly the main concern of the present study is the development of an additional, improved methodology for the use of DLI immunotherapy.

Administration of autologous or allogeneic cytotoxic $\mathrm{T}$ lymphocytes, generated by co-stimulation with dendritic-tumor cell hybrids, tailored to present the putative tumor specific antigens for each individual patient, was already proven to be effective in other malignancies such as prostate cancer and melanoma, as well as in the treatment of post-transplant CMV infections [47-49].

Tumor-specific DLIs are likely to be the most effective method of immunotherapy to achieve a maximal GVL activity. The aim of our study was to induce in vitro a potent donor anti-leukemia cytotoxic $\mathrm{T}$ lymphocyte (CTL) response, utilizing HLA-matched dendritic-leukemia cell hybrids. These leukemia-specific donor CTLs are intended to treat patients with post-transplant relapsed leukemia, after HLA-matched allogeneic stem cell transplantation.

Our results show that such in vitro response is indeed attainable, and that the DC-tumor fusion approach is feasible when the CTLs and the tumor are HLA (A, B, C, DR, DQ) matched. 
Elicitation of CTLs across HLA-matched partners was also demonstrated using DC-tumor cell mixing in vitro without fusion $[15,17]$ or even directly by exposing donor CD8 cells to patient leukemic cells [50]. Nevertheless, it has been strongly suggested by numerous groups that methodology using hybrid cell generation of DCs and tumor cells is more effective in treating malignancies and even metastatic cancer [5,20,51-54].

The stimulatory antigens as well as the target antigens responsible for the CTL induction and activity shown here are largely unknown. We presume that these are tumor specific entities but the involvement of minor histocompatibility antigens can not be excluded. For therapeutic purposes it will be vital to identify the specific CTL clones directed against tumor antigens and "normal" antigens, for example expressed by fibroblasts of the same patient as also suggested by Montagna and Falkenburg [17,50,55]. Characterization of such clones could be then followed by an isolation and expansion of the appropriate CTLs to be injected to patients.

The other alternative would be to administer the DCtumor cell hybrids with or without the "classical" naïve DLI. Injection of such hybrids has already proven effective in certain solid tumors, although in these cases the DCs were either autologous or derived from a third party donor [56] to the tumor.

The validation process of the fusion efficiency was achieved by a number of methodologies to ensure that the PEG mediated fusion generated the appropriate cell composition. This is particularly important since cell aggregates and not genuine fused cells are sometimes induced in the absent of PEG.

It should be noted however that the only way to maximize the probability to obtain the appropriate heterofusion (that is a dendritic cell and a tumor cell) is to start with a pure tumor cell population, although irrelevant fusions of cells cannot be prevented completely. In that respect, Shu et al. have recently formulated a provocative statement about the lack of evidence for heterokaryonic fusion cell formation in various clinical trials and publications. They claim that the evidence for such cell formation was not definitive and this fact resulted maybe in a misconception about the low efficacy of fusion hybrid vaccine. They believe that conclusions drawn from reported clinical trials have not properly evaluated the efficacy of the vaccine and therefore they neither confirm nor disclaim the potential benefits that may be derived from this form of immunotherapy [57].

The CTL activity itself, as assessed by the LDH release assay, was found to be effective and specific, based on a number of experiments performed. It should be noted that we obtained about $30 \%$ killing of the tumor cells. We think that this represents an underestimate of the actual number of the tumor cell eliminated by the CTLs because the LDH assay might not be specific enough, due to the spontaneous release of LDH by the effector cells themselves, especially when high numbers are employed.

In some of the experiments a low but significant rate of tumor cell killing was obtained following the education of CTLs on dendritic cells alone. We speculate that this could be due to the induction of NK activity in the PBL's exposed to autologous dendritic cells. We cannot exclude at this point that the direct exposure of lymphocytes to autologous DC's, may lead to the induction of low non-specific cytotoxic activity. It should be noted however, that the exposure of the same lymphocytes to the tumor cells only, in the same experiments, did not lead to any cytotoxic effect. In this respect, as shown here the co-culture of PBLs with DCs or with tumor cells did not result in the induction of CD8 positive cells as opposed to the high CD8 generation following the coculture of PBLs with the hybrids. No preferential generation of CD4 cells was observed.

Our primary objective was to develop an improved DLI source for active and specific allogeneic cell immunotherapy for post-transplant leukemic relapse in an HLA matched setting. The technology needs to be further developed but the in vitro feasibility demonstrated in this study seems promising.

\section{REFERENCES}

[1] F. I. Alamdari, T. Rasmuson, K. Grankvist, et al., “Angiogenesis and Other Markers for Prediction of Survival in Metastatic Renal Cell Carcinoma," Scandinavian Journal of Urology and Nephrology, Vol. 41, No. 1, 2007, pp. 5-9.

[2] L. Glouchkova, B. Ackermann and D. Dilloo, "Leukemia Vaccines," Acta Haematologica, Vol. 110, No. 4, 2003, pp. 160-170.

[3] M. Robin, M. H. Schlageter, C. Chomienne, et al., "Targeted Immunotherapy in Acute Myeloblastic Leukemia: From Animals to Humans," Cancer Immunology and Immunotherapy, Vol. 54, No. 10, 2005, pp. 933-943.

[4] I. M. Borrello and E. M. Sotomayor, "Cancer Vaccines for Hematologic Malignancies," Cancer Control, Vol. 9, No. 2, 2002, pp. 138-151.

[5] J. Gong, D. Avigan, D. Chen, et al., "Activation of Antitumor Cytotoxic T Lymphocytes by Fusions of Human Dendritic Cells and Breast Carcinoma Cells," Proceedings of National Academy Sciences, Vol. 97, No. 6, 2000(a), pp. 2715-2718.

[6] J. Gong, D. Chen, M. Kashiwaba, et al., "Induction of 
Antitumor Activity by Immunization with Fusions of Dendritic and Carcinoma Cells," Nature Medicine, Vol. 3, No. 4, 1997, pp. 558-561.

[7] M. Klammer, M. Waterfall, K. Samuel, et al., "Fusion Hybrids of Dendritic Cells and Autologous Myeloid Blasts as a Potential Cellular Vaccine for Acute Myeloid Leukaemia," British Journal of Haematology, Vol. 129, No. 3, 2005, pp. 340-349.

[8] R. Ridolfi, L. Ridolfi, M. Petrini, L. Fiammenghi and A. Riccobon, "Dendritic Cell Vaccination and Immunostimulation in Advanced Melanoma," Expert Review of Vaccines, Vol. 2, No. 6, 2003, pp. 825-833.

[9] Y. Becker, "Immunological and Regulatory Functions of Uninfected and Virus Infected Immature and Mature Subtypes of Dendritic Cells - A Review,"Virus Genes, Vol. 26, No. 2, 2003, pp. 119-130.

[10] R. Stripecke, A. M. Levine, V. Pullarkat, et al., "Immunotherapy with Acute Leukemia Cells Modified into Antigen-Presenting Cells: Ex vivo Culture and Gene Transfer Methods," Leukemia, Vol. 16, No. 10, 2002, pp. 19741983.

[11] R. A. Willemsen, C. Ronteltap, P. Chames, et al., "T Cell Retargeting with MHC Class I-Restricted Antibodies: The CD28 Costimulatory Domain Enhances AntigenSpecific Cytotoxicity and Cytokine Production," Journal of Immunology, Vol. 174, No. 12, 2005, pp. 7853-7858.

[12] E. Ranieri, M. Gigante, W. J. Storkus, et al., "Translational Mini-Review Series on Vaccines: Dendritic Cellbased Vaccines in Renal Cancer," Clinical and Experimental Immunology, Vol. 147, No. 3, 2007, pp. 395-400.

[13] J. Galea-Lauri, D. Darling, G. Mufti, et al., "Eliciting Cytotoxic T Lymphocytes against Acute Myeloid Leukemia-Derived Antigens: Evaluation of Dendritic CellLeukemia Cell Hybrids and Other Antigen-Loading Strategies for Dendritic Cell-Based Vaccination," Cancer Immunology and Immunotherapy, Vol. 51, 2002, pp. 299-310.

[14] W. Herr, E. Ranieri, W. Olson, et al., "Mature Dendritic Cells Pulsed with Freeze-Thaw Cell Lysates Define an Effective in Vitro Vaccine Designed to Elicit EBV-Specific CD4(+) and CD8(+) T Lymphocyte Responses," Blood, Vol. 96, No. 5, 2000, pp. 1857-1864.

[15] J. J. Lee, C. E. Nam, J. H. Nam, et al., "Generation of Cytotoxic Donor CD8+ T Cells against Relapsing Leukemic Cells Following Allogeneic Transplantation by Stimulation with Leukemic Cell- or Leukemic Lysate Pulsed Donor Cell-Derived Dendritic Cells," Leukemia Research, Vol. 28, No. 5, 2004, pp. 517-524.

[16] Y. Liu, W. Zhang, T. Chan, et al., "Engineered Fusion Hybrid Vaccine of IL-4 Gene-Modified Myeloma and Relative Mature Dendritic Cells Enhances Antitumor Immunity," Leukemia Research, Vol. 26, No. 8, 2002, pp. 757-763.

[17] D. Montagna, R. Maccario, E. Montini, et al., "Generation and Ex vivo Expansion of Cytotoxic T Lymphocytes Directed toward Different Types of Leukemia or Myelodysplastic Cells Using both HLA-Matched and Partially Matched Donors," Experimental Hematology, Vol. 31, No. 11, 2003, pp. 1031-1038.
[18] W. Song, H. L. Kong, H. Carpenter, et al., "Dendritic Cells Genetically Modified with an Adenovirus Vector Encoding the cDNA for a Model Antigen Induce Protective and Therapeutic Antitumor Immunity," Journal of Experimental Medicine, Vol. 186, No. 8, 1997, pp. 12471256.

[19] D. Avigan, "Dendritic Cells: Development, Function and Potential Use for Cancer Immunotherapy," Blood Review, Vol. 13, No. 1, 1999, pp. 51-64.

[20] J. Gong, N. Nikrui, D. Chen, et al., "Fusions of Human Ovarian Carcinoma Cells with Autologous or Allogeneic Dendritic Cells Induce Antitumor Immunity," Journal of Immunology, Vol. 165, No. 3, 2000(b), pp. 1705-1711.

[21] A. Nencioni and P. Brossart, "Cellular Immunotherapy with Dendritic Cells in Cancer: Current Status," Stem Cells, Vol. 22, No. 4, 2004, pp. 501-513.

[22] S. W. Krause, C. Neumann, A. Soruri, et al., "The Treatment of Patients with Disseminated Malignant Melanoma by Vaccination with Autologous Cell Hybrids of Tumor Cells and Dendritic Cells," Journal of Immunotherapy, Vol. 25, No. 5, 2002, pp. 421-428.

[23] D. K. Schui, L. Singh, B. Schneider, et al., "Inhibiting Effects on the Induction of Cytotoxic T Lymphocytes by Dendritic Cells Pulsed with Lysates from Acute Myeloid Leukemia Blasts," Leukemia Research, Vol. 26, No. 4, 2002, pp. 383-389.

[24] L. M. Faber, S. A. van Luxemburg-Heijs, W. F. Veenhof, et al., "Generation of CD4+ Cytotoxic T-Lymphocyte Clones from a Patient with Severe Graft-Versus-Host Disease after Allogeneic Bone Marrow Transplantation: Implications for Graft-Versus-Leukemia Reactivity," Blood, Vol. 86, No. 7, 1995, pp. 2821-2828.

[25] H. J. Kolb, J. Mittermuller, C. Clemm, et al., "Donor Leukocyte Transfusions for Treatment of Recurrent Chronic Myelogenous Leukemia in Marrow Transplant Patients," Blood, Vol. 76, No. 12, 1990, pp. 2462-2465.

[26] P. A. McSweeney, D. Niederwieser, J. A. Shizuru, et al., "Hematopoietic Cell Transplantation in Older Patients with Hematologic Malignancies: Replacing High-Dose Cytotoxic Therapy with Graft-Versus-Tumor Effects," Blood, Vol. 97, No. 11, 2001, pp. 3390-3400.

[27] T. Mutis, R. Verdijk, E. Schrama, et al., "Feasibility of Immunotherapy of Relapsed Leukemia with Ex VivoGenerated Cytotoxic T Lymphocytes Specific for Hematopoietic System-Restricted Minor Histocompatibility Antigens," Blood, Vol. 93, No. 7, 1999, pp. 2336-2341.

[28] E. Naparstek, A. Nagler, R. Or, et al., "Allogeneic Cellmediated Immunotherapy Using Donor Lymphocytes for Prevention of Relapse in Patients Treated with Allogeneic Bone Marrow Transplantation for Hematological Malignancies," Clinical Transplantation, 1996, pp. 281-290.

[29] E. Naparstek, R. Or, A. Nagler, et al., "T-Cell-Depleted Allogeneic Bone Marrow Transplantation for Acute Leukaemia Using Campath-1 Antibodies and Post-Transplant Administration of Donor's Peripheral Blood Lymphocytes for Prevention of Relapse," British Journal of Haematology, Vol. 89, No. 3, 1995, pp. 506-515. 
[30] S. Slavin, E. Naparstek, A. Nagler, et al., "Allogeneic Cell Therapy with Donor Peripheral Blood Cells and Recombinant Human Interleukin-2 to Treat Leukemia Relapse after Allogeneic Bone Marrow Transplantation," Blood, Vol. 87, No. 6, 1996(a), pp. 2195-2204.

[31] S. Slavin, E. Naparstek, A. Nagler, et al., "Allogeneic Cell Therapy: The Treatment of Choice for All Hematologic Malignancies Relapsing Post BMT," Blood, Vol. 87, No. 9, 1996(b), pp. 4011-4013.

[32] R. J. Soiffer, "Donor Lymphocyte Infusions for Acute Myeloid Leukaemia," Best Practice \& Research Clinical Haematology, Vol. 21, No. 3, 2008, pp. 455-466.

[33] M. Di Nicola, R. Zappasodi, C. Carlo-Stella, et al., "Vaccination with Autologous Tumor-Loaded Dendritic Cells Induces Clinical and Immunologic Responses in Indolent B-Cell Lymphoma Patients with Relapsed and Measurable Disease: A Pilot Study," Blood, Vol. 113, No. 1, 2009, pp. 18-27.

[34] B. Vasir, V. Borges, Z. Wu, et al., "Fusion of Dendritic Cells with Multiple Myeloma Cells Results in Maturation and Enhanced Antigen Presentation," British Journal of Haematology, Vol. 129, No. 5, 2005, pp. 687-700.

[35] J. O. Cullis, Y. Z. Jiang, A. P. Schwarer, et al., "Donor Leukocyte Infusions for Chronic Myeloid Leukemia in Relapse after Allogeneic Bone Marrow Transplantation," Blood, Vol. 79, No. 5, 1992, pp. 1379-1381.

[36] W. R. Drobyski, C. A. Keever, M. S. Roth, et al., "Salvage Immunotherapy Using Donor Leukocyte Infusions as Treatment for Relapsed Chronic Myelogenous Leukemia after Allogeneic Bone Marrow Transplantation: Efficacy and Toxicity of a Defined T-Cell Dose," Blood, Vol. 82, No. 8, 1993, pp. 2310-2318.

[37] D. L. Porter, M. S. Roth, S. J. Lee, et al., "Adoptive Immunotherapy with Donor Mononuclear Cell Infusions to Treat Relapse of Acute Leukemia or Myelodysplasia after Allogeneic Bone Marrow Transplantation," Bone Marrow Transplant, Vol. 18, No. 16, 1996, pp. 975-980.

[38] R. Ivanov, A. Hagenbeek and S. Ebeling, "Towards Immunogene Therapy of Hematological Malignancies," Experimental Hematology, Vol. 34, No. 3, 2006, pp. 251263.

[39] B. D. Johnson, E. E. Becker and R. L. Truitt, "Graftvs.-Host and Graft-vs.-Leukemia Reactions after Delayed Infusions of Donor T-Subsets," Biology of Blood and Marrow Transplantation, Vol. 5, No. 3, 1999, pp. 123-132.

[40] G. Xia, R. L. Truitt and B. D. Johnson, "Graft-versusLeukemia and Graft-versus-Host Reactions after Donor Lymphocyte Infusion are Initiated by Host-Type Antigen-Presenting Cells and Regulated by Regulatory $\mathrm{T}$ Cells in Early and Long-Term Chimeras," Biology of Blood and Marrow Transplantation, Vol. 12, No. 4, 2006, pp. 397- 407.

[41] E. P. Alyea, C. Canning, D. Neuberg, et al., "CD8+ Cell Depletion of Donor Lymphocyte Infusions Using CD8 Monoclonal Antibody-Coated High-Density Microparticles (CD8-HDM) after Allogeneic Hematopoietic Stem Cell Transplantation: A Pilot Study," Bone Marrow Transplant, Vol. 34, No. 2, 2004, pp. 123-128.
[42] R. J. Soiffer, E. P. Alyea, E. Hochberg, et al., "Randomized Trial of CD8+ T-Cell Depletion in the Prevention of Graft-versus-Host Disease Associated with Donor Lymphocyte Infusion," Biology of Blood and Marrow Transplantation, Vol. 8, No. 11, 2002, pp. 625-632.

[43] D. L. Porter, B. L. Levine, N. Bunin, et al., "A Phase 1 Trial of Donor Lymphocyte Infusions Expanded and Activated ex vivo via CD3/CD28 Costimulation," Blood, Vol. 107, No. 4, 2006, pp. 1325-1331.

[44] C. Bonini, G. Ferrari, S. Verzeletti, et al., "HSV-TK Gene Transfer into Donor Lymphocytes for Control of Allogeneic Graft-versus-Leukemia," Science, Vol. 276, No. 5391, 1997, pp. 1719-1724.

[45] K. C. Hsu, C. A. Keever-Taylor, A. Wilton, et al., "Improved Outcome in HLA-Identical Sibling Hematopoietic Stem-Cell Transplantation for Acute Myelogenous Leukemia Predicted by KIR and HLA Genotypes," Blood, Vol. 105, No. 12, 2005, pp. 4878-4884.

[46] L. Ruggeri, M. Capanni, E. Urbani, et al., "Effectiveness of Donor Natural Killer Cell Alloreactivity in Mismatched Hematopoietic Transplants," Science, Vol. 295, No. 5562, 2002, pp. 2097-2100.

[47] B. Carlsson, W. S. Cheng, T. H. Totterman, et al., "Ex vivo Stimulation of Cytomegalovirus (CMV)-Specific $\mathrm{T}$ Cells Using CMV pp65-Modified Dendritic Cells as Stimulators," British Journal of Haematology, Vol. 121, No. 3, 2003, pp. 428-438.

[48] S. El Marsafy and M. Bagot, A. Bensussan, et al., "Dendritic Cells in the Skin-Potential Use for Melanoma Treatment," Pigment Cell and Melanoma Research, Vol. 22, No. 1, 2009, pp. 30-41.

[49] T. J. Lehrfeld, D. I. Lee, "Dendritic Cell Vaccines for the Treatment of Prostate Cancer," Urology and Nephrology, Vol. 26, No. 1, 2008, pp. 576-580.

[50] J. H. Falkenburg, "Immunotherapy of Hematological Malignancies with Dendritic Cells," Hematology Journal, Vol. 5, No. (Suppl. 3), 2004, pp. S96-S99.

[51] W. Guo, Y. Guo, S. Tang, et al., "Dendritic Cell-Ewing's Sarcoma Cell Hybrids Enhance Antitumor Immunity," Clinical Orthopaedics and Related Research, Vol. 466, No. 9, 2008, pp. 2176-2183.

[52] K. Imura, Y. Ueda, T. Hayashi, et al., "Induction of Cytotoxic T Lymphocytes against Human Cancer Cell Lines Using Dendritic Cell-Tumor Cell Hybrids Generated by a Newly Developed Electrofusion Technique," International Journal of Oncology, Vol. 29, No. 3, 2006, pp. 531-539.

[53] R. Savai, R. T. Schermuly, S. S. Pullamsetti, et al., "A Combination Hybrid-Based Vaccination/Adoptive Cellular Therapy to Prevent Tumor Growth by Involvement of T Cells," Cancer Research, Vol. 67, No. 11, 2007, pp. 5443-5453.

[54] T. Yasuda, T. Kamigaki, T. Nakamura, et al., "Dendritic Cell-Tumor Cell Hybrids Enhance the Induction of Cytotoxic T Lymphocytes against Murine Colon Cancer: A Comparative Analysis of Antigen Loading Methods for the Vaccination of Immunotherapeutic Dendritic Cells," Oncology Reports, Vol. 16, No. 6, 2006, pp. 1317-1324. 
[55] J. H. Falkenburg, A. R. Wafelman, P. Joosten, et al., "Complete Remission of Accelerated Phase Chronic Myeloid Leukemia by Treatment with Leukemia-Reactive Cytotoxic T Lymphocytes," Blood, Vol. 94, No. 4, 1999, pp. 1201-1208.

[56] D. Avigan, B. Vasir, J. Gong, et al., "Fusion Cell Vaccination of Patients with Metastatic Breast and Renal Can- cer Induces Immunological and Clinical Responses," Clin Cancer Research, Vol. 10, No. 14, 2004, pp. 4699-4708.

[57] S. Shu, R. Zheng, W. T. Lee, et al., "Immunogenicity of Dendritic-Tumor Fusion Hybrids and their Utility in Cancer Immunotherapy," Critical Reviews in Immunology, Vol. 27, No. 5, 2007, pp. 463-483. 